\title{
ASPECTOS ESTRUCTURALES, COMPOSICIÓN FLORÍSTICA Y CARACTERIZACIÓN ECOLÓGICA DEL BOSQUE DE OYAMEL de la Sierra de Zapalinamé, Coahuila, México
}

\author{
Juan A. Encina-Domínguez ${ }^{1,3}$, Francisco J. Encina-Domínguez ${ }^{1}$, Efrén Mata-Rocha ${ }^{1}$ \\ Y JESÚS VALDES-REYNA ${ }^{2}$
}

'Departamento Forestal, Laboratorio de Sistemas de Información Geográfica, Universidad Autónoma Agraria Antonio Narro, Buenavista, Saltillo 25315, Coahuila, México.

${ }^{2}$ Departamento de Botánica, Universidad Autónoma Agraria Antonio Narro,

Buenavista, Saltillo 25315, Coahuila, México.

${ }^{3}$ Autor para la correspondencia. Correo-e: juanencina@gmail.com

\begin{abstract}
Resumen: La sierra de Zapalinamé se localiza en el sureste de Coahuila, en la transición entre el Desierto Chihuahuense y la Sierra Madre Oriental, es una zona sujeta a conservación ecológica por decreto del gobierno estatal; el matorral xerófilo es la vegetación dominante, sin embargo, en sitios donde el clima es templado frío se desarrolla el bosque de oyamel. Con la finalidad de conocer su composición florística, estructura, además de aspectos sobre su ecología, se establecieron 10 sitios circulares de $1,000 \mathrm{~m}^{2}$ donde se cuantificó el diámetro a $1.30 \mathrm{~m}$ y altura media de las especies arbóreas, además de registrar los arbustos y herbáceas; la regeneración se evaluó en cinco parcelas de $2 \mathrm{~m}^{2}$ por sitio. Se calculó densidad, área basal y valor de importancia de las especies arbóreas. El bosque de oyamel de la sierra de Zapalinamé se desarrolla entre 2,668 - 3,025 m, con 560 - $600 \mathrm{~mm}$ anuales de precipitación y una temperatura media de $12.5^{\circ} \mathrm{C}$, sobre suelos de tipo litosol y con una pendiente entre $34-70 \%$. Está dominado por Abies vejarii, con una alta densidad sobre todo en sitios con mayor altitud, otra especie importante es Pseudotsuga menziesii. Se detectaron plántulas pequeñas, sin embargo, debido al microclima y al suelo, la regeneración es escasa. Este bosque tiene una riqueza de 73 especies. Las familias más numerosas son Asteraceae (15 especies), Pinaceae (4) y Poaceae (4). La conservación del bosque de oyamel es importante por su valor ecológico y por la presencia de especies como Abies vejarii, la cual está listada bajo estatus de conservación, así como por la presencia de varias especies endémicas.
\end{abstract}

Palabras clave: Abies, cerro El Penitente, gradiente de altitud, Pseudotsuga, regeneración.

Abstract: The sierra de Zapalinamé lies at the southeastern portion of the State of Coahuila, in a transitional area between the Chihuahuan Desert Region and the Sierra Madre Oriental. It has the status of a protected natural area by the state government. The Chihuahuan desert scrub is the most common vegetation type; however, spruce forest grows where the climate conditions are cold and temperate. Ten circular plots of $1,000 \mathrm{~m}^{2}$ were established to determine the floristic composition, structure of the forest and other ecological variables. Diameter and height were measured for every tree and shrubs and herbs were recorded. Seedlings were evaluated in five plots of $2 \mathrm{~m}^{2}$ per site. Attributes of the vegetation like density, basal area and the importance value of the tree species were estimated. The spruce forest of the sierra de Zapalinamé is found between 2,668 to 3,025 m of elevation where the annual average precipitation rain varies from 560 to $600 \mathrm{~mm}$ and with a temperature of $12.5^{\circ} \mathrm{C}$, over litosol soils with a slope of $34 \%$ to $70 \%$. The forest is dominated by Abies vejarii, species which grows at elevated places and another important species is Pseudotsuga menziesii. Regeneration is of a few seedlings; nevertheless, due to microclime and soil some regeneration is successful. A total 73 species were recorded in these forests, being the most diverse Asteraceae (15 species), Pinaceae (4) and Poaceae (4). Conservation of the spruce forest is important due to its ecological value; in addition Abies vejarii is listed under status of conservation in the Mexican red list, and also because a number endemic species of plants are in this protected area.

Key words: Abies, altitudinal gradient, cerro El Penitente, Pseudotsuga, regeneration.

os bosques de oyamel son comunidades vegetales integradas por Abies, Pseudotsuga y Picea, en México cubren aproximadamente 32,000 hectáreas, siendo Abies religiosa y Pseudotsuga menziesii var. glauca las especies más comunes (Rzedowski, 1978). Estos bosques están con- finados a laderas protegidas del viento así como de la intensidad solar fuerte, muchas veces en cañadas o barrancas que ofrecen un microclima especial (Rzedowski, 1978) ó en terrenos con fuertes pendientes (Madrigal, 1967). Los bosques de mayor extensión se presentan en la Faja Vol- 
ENCINA-DOMÍNGUEZ, ET AL.

cánica Transmexicana, así como en la Sierra Madre del Sur, comunidades importantes se localizan en Guerrero y al sur de Oaxaca (Rzedowski, 1978). En Coahuila, VillarrealQuintanilla y Valdés-Reyna (1992-93) mencionan bosques de oyamel para la sierra de Arteaga, el Jabalí, de la Madera y del Carmen. En la sierra de Zapalinamé, Arce y Marroquín (1985) reportan para el cerro El Penitente un bosque con Pseudotsuga menziesii, Cupressus arizonica y Abies vejarii, otras especies arbóreas son Pinus hartwegii y P. greggii, además de un estrato arbóreo inferior de Arbutus xalapensis, Quercus greggii y Prunus serotina.

En México la superficie forestal se ha reducido progresivamente, originando problemas ecológicos, económicos y sociales. Entre los factores de mayor degradación se mencionan aprovechamientos forestales mal planeados, modificación en el régimen del fuego, pastoreo y contaminación atmosférica (Bauer y Hernández, 1986; Alvarado et al. 1991; López-López et al. 1998; Saavedra et al., 2003), así como la fuerte presión por el avance de la frontera agrícola, la mancha urbana, plagas y enfermedades (Verduzco-Gutiérrez, 1976). La sierra de Zapalinamé es una zona sujeta a conservación ecológica, decretada como Área Natural Protegida por el gobierno de Coahuila (Anónimo, 1996), ocupando una superficie de casi 45,000 hectáreas (Anónimo, 1998). Actualmente los bosques de oyamel de este macizo montañoso no presentan impactos antropogénicos, debido a su ubicación inaccesible, sin embargo, considerando que los incendios forestales son frecuentes en el área surge la preocupación por su conservación. La presente investigación pretende determinar la estructura y composición de especies leñosas que integran el bosque de oyamel del cerro El Penitente en la sierra de Zapalinamé, además de su distribución, aspectos ecológicos y especies asociadas.

\section{Área de estudio}

La sierra de Zapalinamé se localiza en el sureste de Coahuila, en los municipios de Saltillo y Arteaga; es una zona de transición entre el Desierto Chihuahuense y la Sierra Madre Oriental. Se ubica entre $25^{\circ} 15^{\prime} 00^{\prime \prime}-25^{\circ} 25^{\prime} 58.35^{\prime \prime}$ de latitud Norte y entre $100^{\circ} 47^{\prime} 14.5^{\prime \prime}-101^{\circ} 05^{\prime} 3.8^{\prime \prime}$ de longitud Oeste (figura 1). Pertenece a la subprovincia de la Gran Sierra Plegada; el macizo incluye valles, planicies y elevaciones plegadas (Anónimo, 1998).

La orientación de los pliegues transversales es de este a oeste, con altitudes entre 1,590 a 2,200 m, alcanzando su mayor elevación a 3,140 m en el cerro El Penitente. Las rocas del área son sedimentarias marinas del Jurásico y Cretácico; las calizas ocupan $43 \%$ del área, las areniscas y conglomerados $17 \%$. Los suelos de tipo litosol ocupan el $49 \%$ y las rendzinas representan el 29\%. El área tiene un clima BSokw"(e), seco, templado, extremoso en las partes bajas de la zona y templado C(wo) en las altas, hacia la parte sur del macizo montañoso el clima es semiárido, llegando a ser subhúmedo en el sureste, la temperatura media anual es de $18^{\circ} \mathrm{C}$ (Meganck et al., 1981). La precipitación promedio es $498 \mathrm{~mm}$, de tipo convectivo y ocurre durante el verano (Anónimo, 1983). Las áreas con exposición sur están cubiertas por matorral rosetófilo y micrófilo, mientras que en partes altas la vegetación es bosque de pino y oyamel; en los cañones se presentan bosques de encino y en las laderas bajas de exposición norte y oeste se distribuye el matorral submontano de rosáceas (Marroquín, 1976). Los tipos de vegetación más extensos son el bosque de pino, bosque de piñonero y bosque de piñonero con matorral xerófilo, distribuidos en el $36.2 \%$ del área protegida, mientras que el bosque de oyamel ocupa únicamente el $1.86 \%$ y está dominado por Pseudotsuga menziesii y Abies vejarii, los arbustos más frecuentes son Symphoricarpos microphyllus, Paxistima myrsinites y Quercus hintoniorum, en el estrato herbáceo dominan Bromus carinatus, Ageratina calophylla, Pleopeltis guttata y Thalictrum grandidentatum.

\section{Metodología}

Se establecieron 10 sitios de muestreo circulares de 1,000 $\mathrm{m}^{2}$ en el bosque de oyamel del cerro El Penitente, mediante la compensación por pendiente propuesta por Medina (1983) y ubicados a una equidistancia entre sitios de $200 \mathrm{~m}$, a través de un gradiente altitudinal. Para la medición de la vegetación se utilizó el método de parcela (Mueller-Dombois y Ellemberg, 1974) donde se cuantificó el diámetro normal (a $1.30 \mathrm{~m}$ ) de las especies arbóreas (individuos con diámetro $\geq 5 \mathrm{~cm}$ ), así como la altura media, además de registrar los arbustos y herbáceas; para evaluar la regeneración de las especies arbóreas (individuos $\leq 1.30 \mathrm{~m}$ de altura) se establecieron cinco cuadrantes de $2 \mathrm{~m}^{2}$ (uno al centro y cuatro en los puntos cardinales de cada sitio de muestreo (Olvera et al., 1996; Figueroa-Rangel y Olvera-Vargas, 2000; ÁvilaBello y López-Mata, 2001; Galindo-Jaimes et al., 2002). En cada sitio se registró altitud (tomada de un altímetro-barómetro), pendiente (mediante una pistola Haga) y exposición topográfica (tomada con una brújula). Se calcularon los atributos de la vegetación y con la suma de los valores relativos se obtuvo el valor de importancia relativa por especie (Mueller-Dombois y Ellenberg, 1974). La estructura horizontal cuantitativa se analizó en términos del área basal y de la densidad de árboles en las categorías diamétricas mayores a $5 \mathrm{~cm}$ de diámetro normal. Para determinar la dominancia estructural de Abies vejarii a través del gradiente de altitud, se efectuó una regresión lineal simple con datos sobre densidad y altitud. Las variables ecológicas de temperatura y precipitación para cada sitio de muestreo se obtuvieron por interpolación de la información proveniente del análisis de regresión múltiple, con datos de los últimos 10 años, registrados en las estaciones meteorológicas cercanas al área de estudio, se utilizaron las variables altitud en base al modelo digital del terreno (variable 1), latitud (variable 2), longitud 

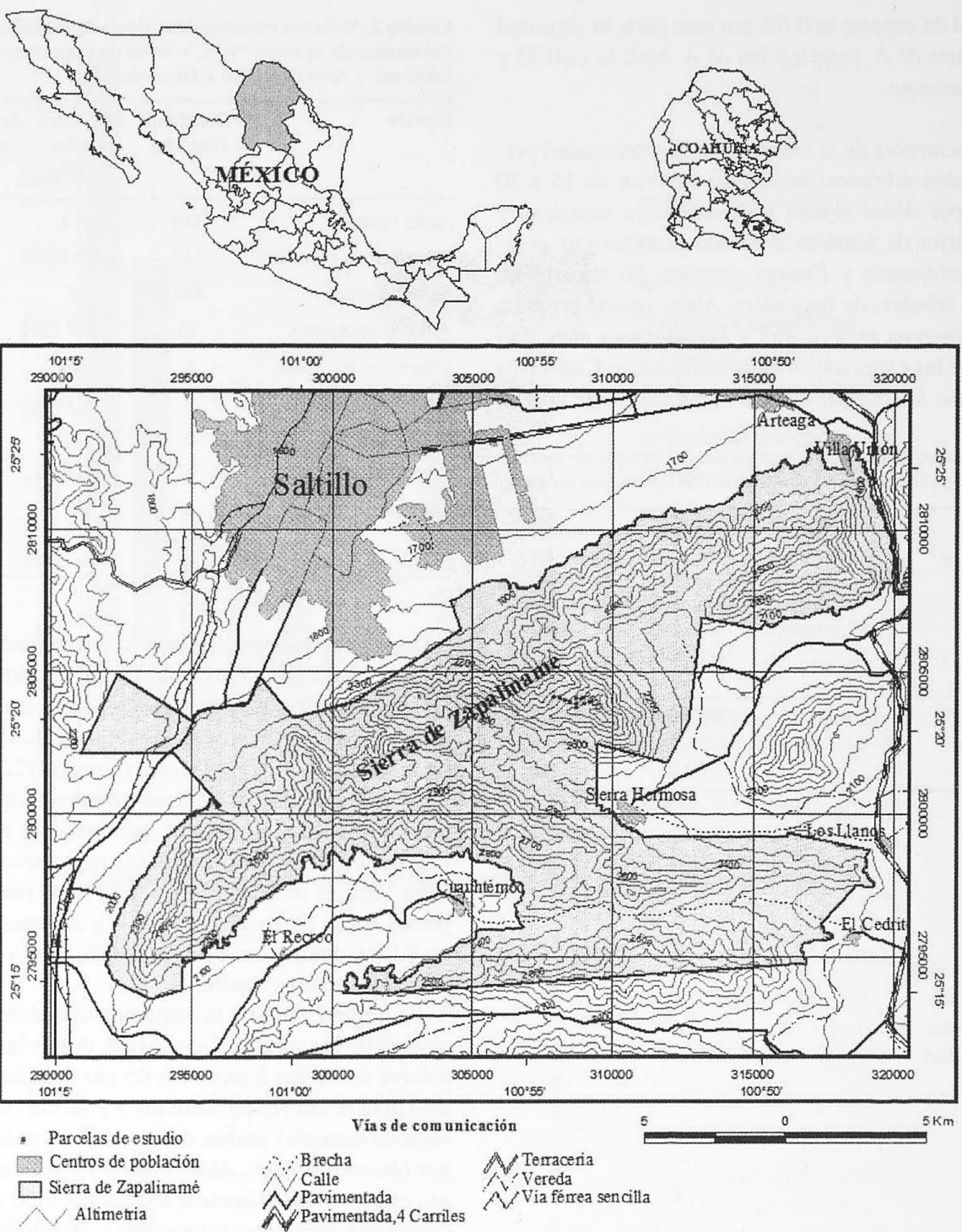

Figura 1. Localización de la sierra de Zapalinamé y ubicación de las parcelas de estudio en el bosque de oyamel.

(variable 3) para obtener un modelo digital de los valores medios anuales de precipitación y temperatura. Se realizó un análisis de la composición florística del bosque con otros bosques de oyamel, mediante el índice de similitud de Jaccard (Mueller-Dombois y Ellenberg, 1974).

\section{Resultados}

Composición florística. La flora vascular está integrada por 37 familias, 60 géneros y 73 especies, las familias con mayor riqueza son: Asteraceae (15 especies), Poaceae (5), Pinaceae (4), y Lamiaceae (4), los géneros con más espe- cies son: Senecio (4), Ageratina (4), Quercus (3), Salvia (3), Pinus (2), Arenaria (2) y Euphorbia (2) (ver listado florístico en Anexo 1). Del total de las especies registradas las dicotiledóneas representan el $75.3 \%$, las monocotiledóneas el $12.3 \%$ y el $12.3 \%$ pertenecen a las divisiones Pteridophyta y Pinophyta. En el cuadro 1, se compara la composición de los bosques de Abies vejarii, A. religiosa y $A$. hickelii, además de algunos aspectos ecológicos de estas comunidades; la lista de especies incluye a 28 taxa (17 árboles, 2 arbustos y 9 hierbas), los bosques presentan seis géneros y tres especies en común; la similitud a nivel de género entre los bosques de $A$. vejarii y los de $A$. religiosa, es 0.94 mien- 
tras que a nivel de especie es 0.69 , por otra parte la similitud entre los bosques de A. vejarii y los de A. hickelii es 0.33 y 0.15 respectivamente.

Aspectos estructurales de la vegetación. La comunidad presenta dos estratos arbóreos: un estrato superior de 15 a 20 $\mathrm{m}$, integrado por Abies vejarii y Pseudotsuga menziesii y un estrato inferior de 4 a $6 \mathrm{~m}$, formado por Quercus greggii, Arbutus xalapensis y Prunus serotina. Se registró un total de 1,174 árboles, de los cuales, Abies vejarii presenta 439 ind./ha, Quercus greggii 463 y Pseudotsuga menziesii 232, el resto de las especies: Arbutus xalapensis, Cupressus arizonica, Pinus hartwegii, Prunus serotina, Pinus greggii

Cuadro 1. Comparación entre la composición del bosque de oyamel de la sierra de Zapalinamé con otros bosques de oyamel en México.

\begin{tabular}{|c|c|c|c|}
\hline & $\begin{array}{c}\text { Bosque } \\
\text { de Abies } \\
\text { vejarii } \\
\text { (este } \\
\text { estudio) }\end{array}$ & $\begin{array}{c}\text { Bosque } \\
\text { de Abies } \\
\text { religiosa } \\
\text { (Rzedowski } \\
\text { et al, 2001, } \\
\text { Sánchez et al, } \\
\text { 2005) }\end{array}$ & $\begin{array}{c}\text { Bosque } \\
\text { de } A b i e s \\
\text { hickelii } \\
\text { (Ávila-Bello, } \\
\text { 2000) }\end{array}$ \\
\hline $\begin{array}{l}\text { Altitud, precipitación, } \\
\text { temperatura media }\end{array}$ & $\begin{array}{l}300-3100 \mathrm{~m}, \\
590 \mathrm{~mm} \\
12.5^{\circ} \mathrm{C}\end{array}$ & $\begin{array}{c}2700-3500 \mathrm{~m} \\
1200 \mathrm{~mm} \\
10.5^{\circ} \mathrm{C}\end{array}$ & $\begin{array}{c}3000-3600 \mathrm{~m}, \\
1698 \mathrm{~mm}, \\
9.3^{\circ} \mathrm{C}\end{array}$ \\
\hline Zona geográfica & $\begin{array}{l}\text { apalinamé, } \\
\text { Coahuila }\end{array}$ & $\begin{array}{c}\text { Faja } \\
\text { Volcánica } \\
\text { Transmexicana }\end{array}$ & $\begin{array}{l}\text { Pico de } \\
\text { Orizaba, } \\
\text { Veracruz }\end{array}$ \\
\hline Abies vejarii & $x$ & & \\
\hline Abies religiosa & & $x$ & $x$ \\
\hline Abies hickelii & & & $x$ \\
\hline Pseudotsuga menziesii & $x$ & $x$ & \\
\hline Cupressus arizonica & $x$ & & \\
\hline Cupressus lusitanica & & $x$ & \\
\hline Pinus pseudostrobus & & & $x$ \\
\hline Pinus ayacahuite & & & $x$ \\
\hline Pinus patula & & & $x$ \\
\hline Pinus hatwegii & $x$ & $x$ & \\
\hline Quercus greggii & $x$ & & $x$ \\
\hline Quercus laurina & & $x$ & $x$ \\
\hline Salix oxylepis & & & $x$ \\
\hline Salx paradoxa & $x$ & $x$ & $x$ \\
\hline Alnus jorullensis & & $x$ & \\
\hline Arbutus xalapensis & $x$ & $x$ & $x$ \\
\hline Prunus serotina & $x$ & $x$ & \\
\hline Symphoricarpos microphyllus & $x$ & $x$ & \\
\hline Lonicera pilosa & $x$ & $x$ & \\
\hline Echeandia flavescens & $x$ & $x$ & \\
\hline Nectouxia formosa & $x$ & $x$ & \\
\hline Achillea millefolium & $x$ & $x$ & \\
\hline Arenaria lanuginosa & $x$ & $x$ & \\
\hline Arenaria lycopodioides & $x$ & $x$ & \\
\hline Brachypodium mexicanum & $x$ & $x$ & \\
\hline Dahlia coccinea & $x$ & $x$ & $x$ \\
\hline Penstemon campanulatus & $x$ & $x$ & \\
\hline Chimaphila umbellata & $x$ & $x$ & \\
\hline
\end{tabular}

Cuadro 2. Atributos estructurales calculados para las especies arbóreas del bosque de oyamel. *V.I.E. = Valor de importancia ecológica (Densidad rel. + Area basal rel. + Frecuencia rel. /3).

\begin{tabular}{lcccc}
\hline Especie & $\begin{array}{c}\text { Densidad } \\
\text { (ind/Ha) }\end{array}$ & $\begin{array}{c}\text { Diámetro } \\
\text { medio } \\
(\mathbf{c m})\end{array}$ & $\begin{array}{c}\text { Área Basal } \\
\left(\mathbf{m}^{2} / \mathbf{H a}\right)\end{array}$ & V.I.E.* \\
\hline Abies vejarii & 439 & 18.3 & 16.44 & 38.41 \\
Pseudotsuga menziesii & 232 & 19.1 & 9.24 & 24.52 \\
Quercus greggii & 463 & 6.7 & 2.02 & 22.97 \\
Arbutus xalapensis & 18 & 9.7 & 0.14 & 4.76 \\
Cupressus arizonica & 6 & 22.5 & 0.77 & 2.89 \\
Pinus hartwegii & 2 & 50.0 & 0.85 & 2.56 \\
Prunus serotina & 6 & 10.0 & 0.05 & 1.76 \\
Pinus greggii & 3 & 25.0 & 0.15 & 1.15 \\
Quercus saltillensis & 5 & 8.0 & 0.03 & 0.98 \\
Total & $\mathbf{1 , 1 7 4}$ & -- & $\mathbf{2 9 . 6 9}$ & $\mathbf{1 0 0}$ \\
\hline
\end{tabular}

y Quercus saltillensis, presentan una densidad inferior a 18 ind./ha (cuadro 2). Los valores de importancia ecológica (V.I.E) más altos están repartidos entre las especies dominantes y corresponden a Abies vejarii (38.41\%), Pseudotsuga menziesii (24.52) y Quercus greggii (22.97), lo cual no ocurre en el análisis del diámetro medio, ya que los diámetros mayores $(50,25,22.50 \mathrm{~cm})$, pertenecen a Pinus hartwegii, P. greggii y Cupressus arizonica respectivamente, especies cuyo V.I.E es de $2.56,1.15$, y $2.89 \%$, se presentan otras especies como Quercus saltillensis y Arbutus xalapensis con un V.I.E y diámetro medio de $0.98 \%, 8 \mathrm{~cm}$ y $4.76 \%, 9.72 \mathrm{~cm}$ respectivamente (cuadro 2).

La figura 2 muestra la estructura diamétrica del bosque de oyamel de la sierra de Zapalinamé, donde la densidad se distribuye desde los 5 hasta los $95 \mathrm{~cm}$ de diámetro. La mayor densidad se concentra entre los 5 y $10 \mathrm{~cm}$ (431y 298 ind./ha respectivamente) ambas dominadas en orden descendiente por Quercus greggii, Abies vejarii y Pseudotsuga menziesii, mientras que en diámetros superiores a $30 \mathrm{~cm}$, la densidad disminuye de manera importante y está dominada por Abies vejarii y Pseudotsuga menziesii.

Las especies del género Pinus ocurren en bajas densidades, así, la participación de Pinus greggii en la estructura del bosque es de 3 ind./ha repartidos entre los 15,20 y 30 $\mathrm{cm}$ de diámetro, mientras que Pinus hartwegii presenta una densidad de 2 ind./ha en las categorías de 40 y $60 \mathrm{~cm}$.

Los resultados muestran una relación inversa entre la densidad y las categorías diamétricas ya que a medida que aumenta la densidad del arbolado disminuye el diámetro de éste (figura 2). La distribución diamétrica de las especies dominantes fisonómicas del bosque de oyamel se presenta en la figura 3, donde se observa una dominancia de Abies vejarii sobre Pseudotsuga menziesii en las categorías inferiores a $40 \mathrm{~cm}$. Como parte del área basal se registró un total de 
ESTRUCTURA Y FLORÍSTICA DEL BOSQUE DE OYAMEL, ZAPALINAMÉ

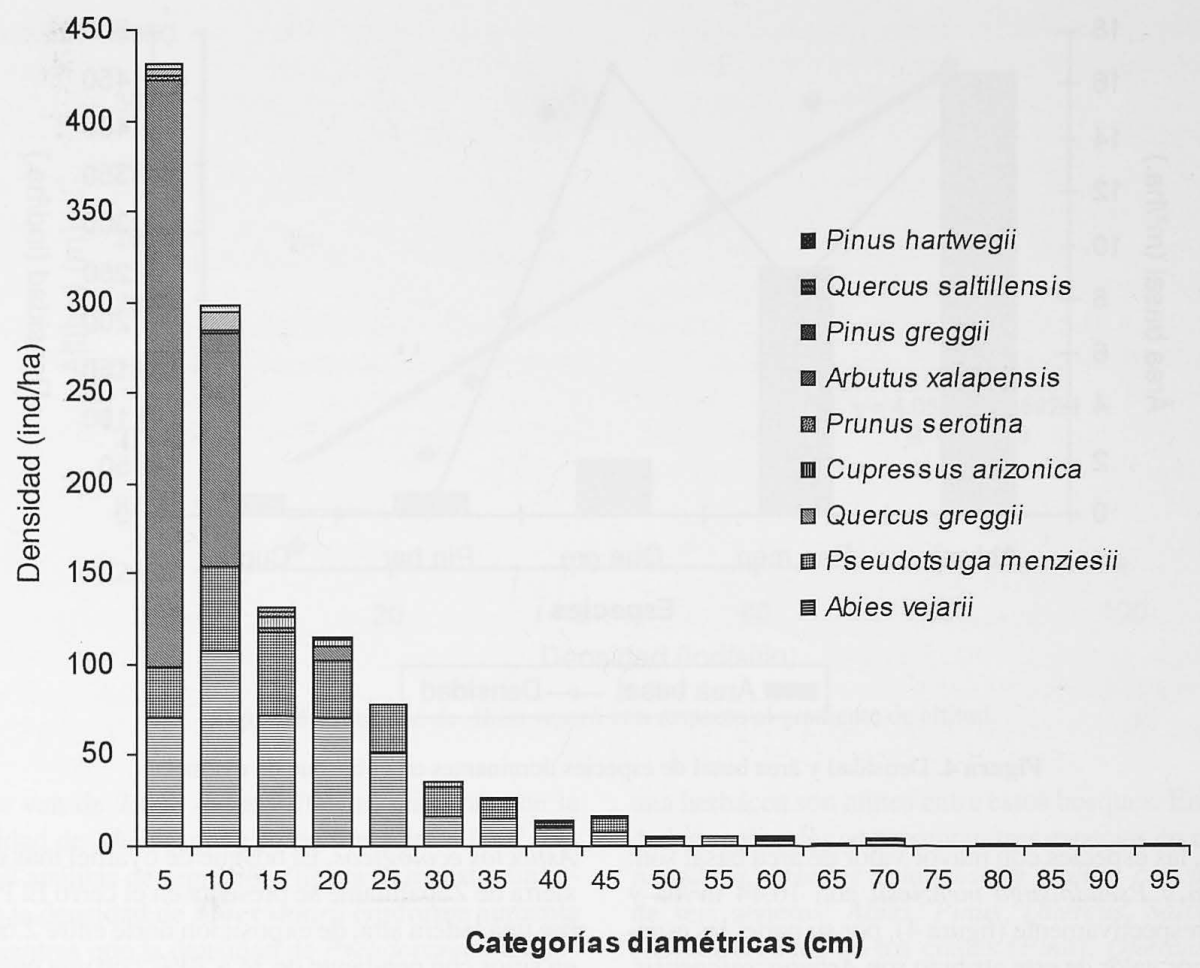

Figura 2. Distribución del arbolado por categorías diamétricas en el bosque de oyamel.

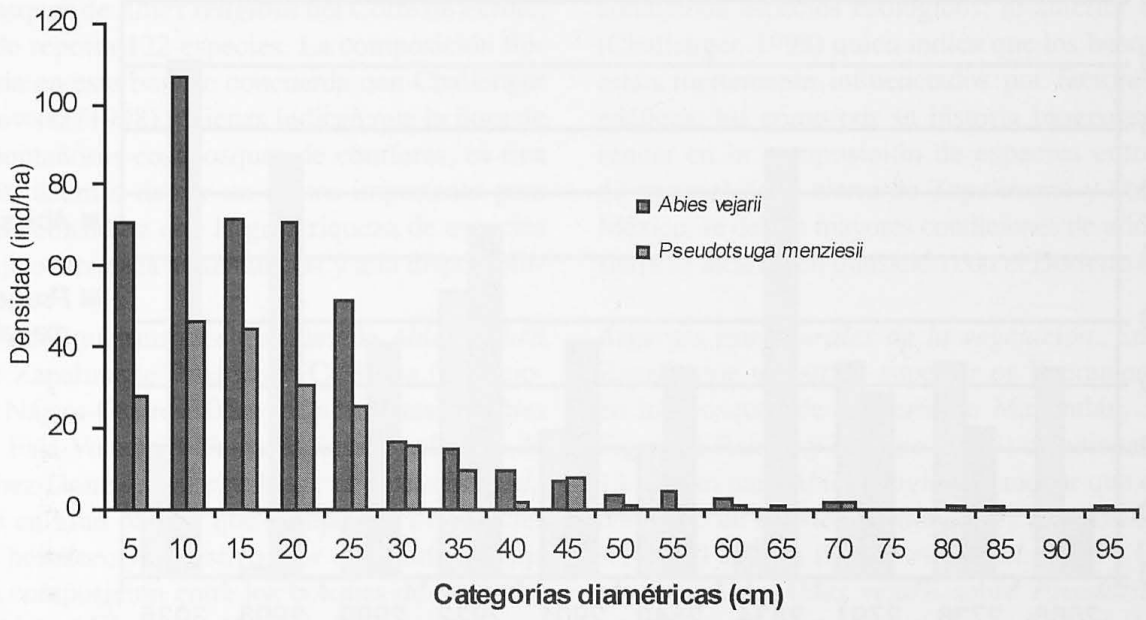

Figura 3. Distribución diamétrica de las especies (Abies vejarii y Pseudotsuga menziesii). 


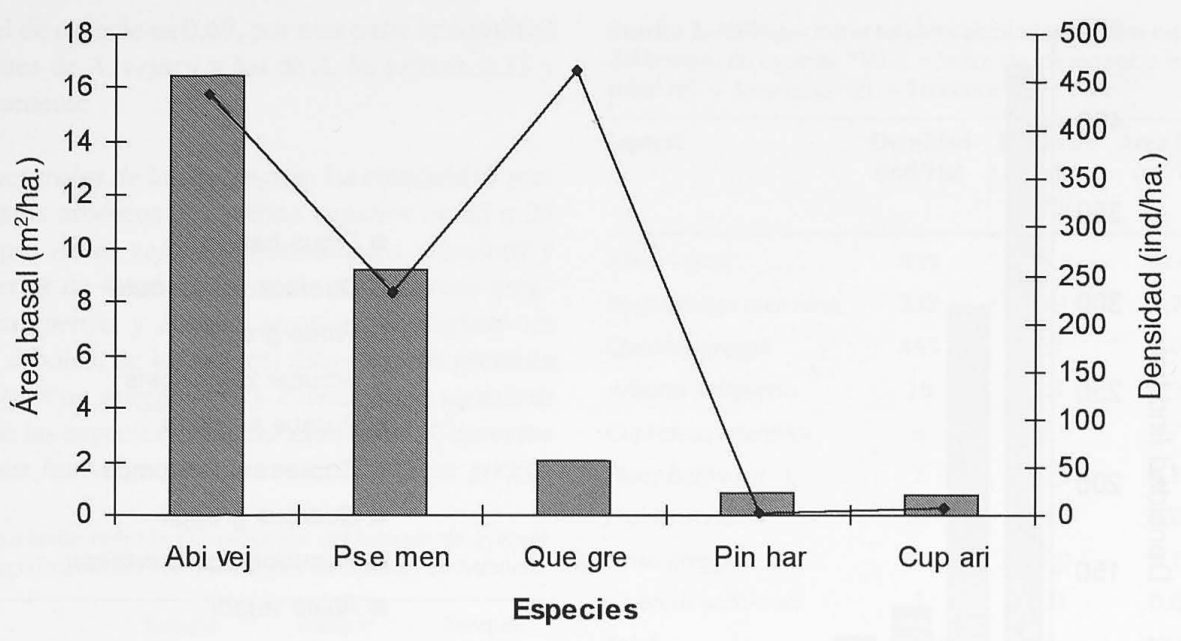

Area basal $\multimap$ Densidad

Figura 4. Densidad y área basal de especies dominantes en el bosque de oyamel.

$29.69 \mathrm{~m}^{2} / \mathrm{ha}$, las especies con mayor valor de área basal son: Abies vejarii y Pseudotsuga menziesii con $16.44 \mathrm{~m}^{2} /$ ha $\mathrm{y}$ $9.24 \mathrm{~m}^{2} /$ ha respectivamente (figura 4), por su parte, las especies con menor valor de este atributo son Arbutus xalapensis, Prunus serotina y Quercus saltillensis con $0.14,0.05$ y 0.03 $\mathrm{m}^{2} /$ ha respectivamente (cuadro 2). La regeneración de Abies vejarii y Pseudotsuga menziesii, está integrada por plántulas con una densidad de 518,340 ind./ha y una altura de $2-4 \mathrm{~cm}$, distribuida de manera irregular y se concentra en la cercanía de los árboles semillero.
Aspectos ecológicos. El bosque de oyamel más extenso de la sierra de Zapalinamé se presenta en el cerro El Penitente, sobre una ladera alta, de exposición norte entre 2,668 - 3,025 m en sitios con pendiente de 34 a $70 \%$, con una precipitación de 560 a $600 \mathrm{~mm}$ anuales y una temperatura media de $12.5^{\circ} \mathrm{C}$ (ver información por sitio de muestreo en el Anexo 2), sobre suelos poco profundos y con abundante materia orgánica. La densidad de Abies vejarii y Pseudotsuga menziesii a través del gradiente altitudinal, se observa en la figura 5, donde Pseudotsuga menziesii presenta mayor densidad en

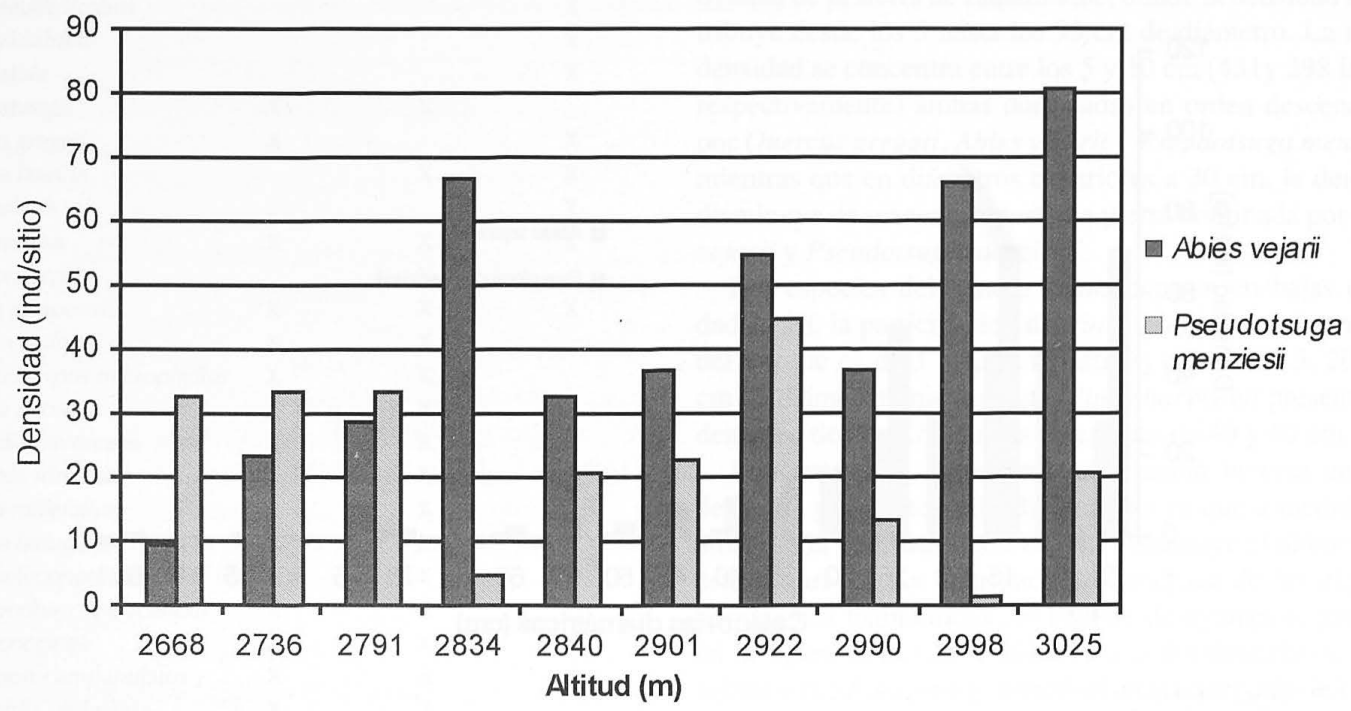

Figura 5. Densidad de Abies vejarii y Pseudotsuga menziesii a través del gradiente de altitud. 


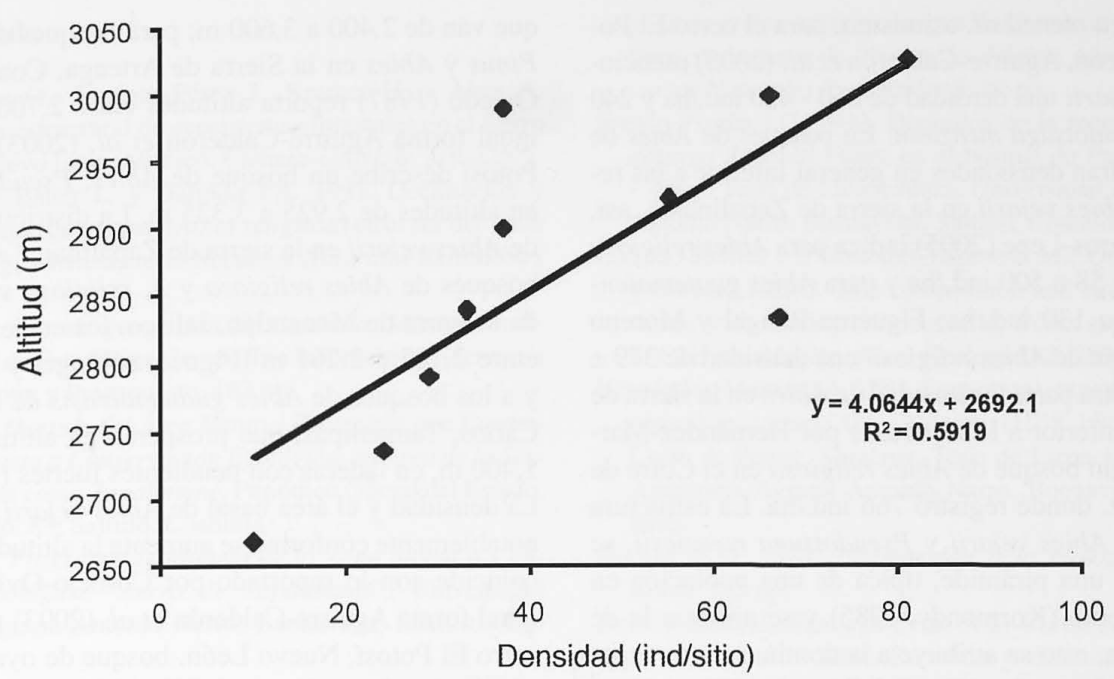

Figura 6. Densidad de Abies vejarii con respecto al gradiente de altitud.

altitudes que van de 2,668 hasta 2,791 m, mientras que la mayor densidad de Abies vejarii se presenta entre los 2,834 y 3,025 m. El análisis de regresión (figura 6) muestra un incremento en la densidad de Abies vejarii conforme aumenta la altitud, mientras que la densidad de Pseudotsuga menziesii disminuye.

\section{Discusión}

Composición florística. La riqueza de especies del bosque de oyamel es mayor a la reportada por Cisneros-Lepe (2005) para bosques de Abies religiosa de Jalisco, quien encontró 64 especies y menor a la registrada por Narave-Flores (1985), para bosques de Abies religiosa del Cofre de Perote, Veracruz, donde reporta 122 especies. La composición florística registrada en este bosque concuerda con Challenger (1998) y Rzedowski (1998), quienes indican que la flora de las regiones montañosas con bosques de coníferas, es rica en endemismos, además de ser un centro importante para muchos taxa. Se considera que la gran riqueza de especies se debe a la baja ocurrencia de disturbios y a la disponibilidad de humedad.

La elevada similitud entre los bosques de Abies vejarii de la sierra de Zapalinamé y Arteaga, Coahuila (CornejoOviedo, 1987; Nájera-Castro, 2000) y los bosques de Abies religiosa de la Faja Volcánica Transmexicana y el Valle de México (Sánchez-González et al., 2005; Rzedowski et al., 2001), se debe en gran parte a que comparten 14 especies de los estratos herbáceo y arbustivo. Por otra parte, la baja similitud en la composición entre los bosques de Abies vejarii y los de Abies hickelii del Cofre de Perote y la sierra de Acultzingo, Veracruz (Ávila-Bello, 2000; Ávila-Bello y López-Mata, 2001), se debe a que tres especies leñosas y una herbácea son afines entre estos bosques. En las comunidades analizadas se presentan tres especies en común: Salix paradoxa, Arbutus xalapensis y Dahlia coccinea, además de seis géneros: Abies, Pinus, Quercus, Salix, Arbutus y Dahlia, algunos de los cuales (Pinus, Quercus y Arbutus) forman parte de la descripción referida por Rzedowski, (1978), para bosques de Abies. El estrato arbóreo de las comunidades analizadas se integra por especies de afinidad boreal (Rzedowski, 1991), al igual que los estratos herbáceo y arbustivo de los boques de A. vejarii y A. religiosa, mientras que los de $A$. hickelii tienen más elementos mexicanosudamericanos (Rzedowski, 1978). La composición de tales bosques está influenciada por su ubicación geográfica, que condiciona aspectos ecológicos; lo anterior concuerda con (Challenger, 1998) quien indica que los bosques templados están fuertemente influenciados por factores climáticos y edáficos, así como por su historia biogeográfica. La diferencia en la composición de especies entre los bosques de oyamel de la sierra de Zapalinamé y otros bosques de México, se debe a mayores condiciones de aridez, ya que ésta sierra se localiza en transición con el Desierto Chihuahuense.

Aspectos estructurales de la vegetación. En el bosque de Zapalinamé el estrato superior es ligeramente mayor que en los bosques de la sierra de Manantlán, Jalisco, donde Figueroa-Rangel y Moreno (1993) mencionan una altura de 13 a $20 \mathrm{~m}$ para Abies religiosa y menor que en los bosques del Valle de México, donde Abies religiosa, forma un dosel de 20 a $40 \mathrm{~m}$ (Rzedowski et al., 2001). La dominancia estructural de Abies vejarii sobre Pseudotsuga menziesii, también se presenta en la sierra de Arteaga, donde CornejoOviedo (1987) y Nájera-Castro (2000) reportan densidades de 225 - 727 ind./ha para Abies vejarii y 120 - 332 ind./ha 
para Pseudotsuga menziesii, asimismo, para el cerro El Potosí en Nuevo León, Aguirre-Calderón et al. (2003) mencionan de Abies vejarii una densidad de 360 - 420 ind./ha y 240 ind./ha para Pseudotsuga menziesii. En bosques de Abies de México, se registran densidades en general inferior a las registradas para Abies vejarii en la sierra de Zapalinamé, así, en Jalisco, Cisneros-Lepe (2005) indica para Abies religiosa una densidad de 58 a 500 ind./ha y para Abies guatemalensis var. jaliscana 130 ind./ha; Figueroa-Rangel y Moreno (1993) mencionan de Abies religiosa una densidad de $379 \pm$ 54 ind./ha. Por otra parte la densidad de Abies en la sierra de Zapalinamé es inferior a lo reportado por Hernández-Martínez (1984) en un bosque de Abies religiosa en el Cofre de Perote, Veracruz, donde registró 766 ind./ha. La estructura diamétrica para Abies vejarii y Pseudotsuga menziesii, se asemeja a la de una pirámide, típica de una población en vías de crecimiento (Kormondy, 1985) y se ajusta a la de una "J" invertida, esto se atribuye a la dominancia de especies tolerantes a la sombra (Spurr y Barnes, 1982) y a que se trata de un bosque incoetáneo (Jardel-Peláez et al., 2004; Daniel et al., 1982). En cuanto a la dominancia en área basal de Abies vejarii sobre Pseudotsuga menziesii, se han registrado datos similares en la sierra de Arteaga, Coahuila, donde Cornejo-Oviedo (1987) y Nájera-Castro (2000) reportan para Abies vejarii $12.08-14.66 \mathrm{~m}^{2} /$ ha y $7.27-7.88$ $\mathrm{m}^{2} /$ ha para Pseudotsuga menziesii, en los bosques del cerro El Potosí en Nuevo León, Aguirre-Calderón, et al. (2003) mencionan que Abies vejarii presenta de 13.7 a $24 \mathrm{~m}^{2} /$ ha $y$ Pseudotsuga menziesii $14 \mathrm{~m}^{2} / \mathrm{h}$. La regeneración establecida es escasa, de acuerdo a los criterios de Spurr y Barnes (1982), Hocker (1984) y Niembro-Rocas (1986), quienes consideran establecidas a las plántulas con un crecimiento vigoroso, tomando un período de 1 a 3 años, esto se atribuye a un incremento en las condiciones de aridez (Anónimo, 1998) y una precipitación baja (590 mm), de acuerdo al régimen de 1000 - $1700 \mathrm{~mm}$ reportada para bosques de Abies en México (Rzedowski, 1978; Ávila-Bello y López-Mata, 2001) se observó que pocos individuos superan el estadio de plántula, debido a que las semillas que germinan en el suelo vegetal durante la estación de lluvias, mueren en los meses subsecuentes por déficit de humedad sin alcanzar el suelo mineral. En bosques de la sierra de Arteaga Coahuila, se reporta una densidad de 2,164 - 4,150 ind./ha para Abies vejarii y 824 - 1,990 ind./ha de Pseudotsuga menziesii, con altura media de 0.83 m (Cornejo-Oviedo, 1987; Franco-Pizaña, 1990; Reyna-Olvera, 1998), el fuerte contraste entre estos valores y los observados en la sierra de Zapalinamé se debe a precipitaciones mayores (924 mm) registradas por NájeraCastro (2000) en la sierra de Arteaga.

Aspectos ecológicos. La distribución altitudinal del bosque de oyamel se ubica dentro del rango de distribución de la mayoría de estas comunidades en México, así, Rzedowski (1978) describe bosques de oyamel en sitios con altitudes que van de 2,400 a 3,600 m; para bosques de Pseudotsuga, Pinus y Abies en la Sierra de Arteaga, Coahuila, CornejoOviedo (1987) reporta altitudes entre 2,700 y 3,360 m, de igual forma Aguirre-Calderón et al. (2003) en el cerro El Potosí describe un bosque de Abies, Pseudotsuga y Pinus en altitudes de 2,925 a 3,325 m. La distribución altitudinal de Abies vejarii en la sierra de Zapalinamé es superior a los bosques de Abies religiosa y A. religiosa var. emarginata, de la sierra de Manantlán, Jalisco, los cuales se desarrollan entre 2,187 a 2,261 m (Figueroa-Rangel y Moreno, 1993) y a los bosques de Abies guatemalensis de la sierra de San Carlos, Tamaulipas, que prosperan en altitudes de 1,300 a 1,400 m, en laderas con pendientes fuertes (Briones, 1991). La densidad y el área basal de Abies vejarii se incrementan notablemente conforme se aumenta la altitud, esta tendencia coincide con lo reportado por Cornejo-Oviedo (1987); de igual forma Aguirre-Calderón et al. (2003) reportan para el cerro El Potosí, Nuevo León, bosque de oyamel con dominancia de Abies vejarii en sitios de 3,300 a 3,600 m de altitud, asimismo, Ávila-Bello y López-Mata (2001) en los bosques de Abies hickelii en México, registraron un incremento en la dominancia de esta especie en sitios con altitudes que oscilan entre los 3,000 y $3,600 \mathrm{~m}$.

El bosque de oyamel de la sierra de Zapalinamé es importante, dado que es el único de su tipo en el área, su distribución se limita a la exposición norte del área más alta de la sierra (cerro El Penitente), donde se presentan condiciones para el bosque de Abies vejarii y Pseudotsuga menziesii var. glauca (Farjon, 1990). Dentro de la riqueza de especies, la de mayor relevancia es Abies vejarii incluida en la NOM059-SEMARNAT-2001 en la categoría de Amenazada (SEMARNAT, 2002), adicionalmente, en este bosque se presentan otras especies como: Quercus saltillensis (Fagaceae), Ageratina zapalinama (Asteraceae) y Festuca valdesii (Poaceae), endémicas para el norte de la Sierra Madre Oriental en Coahuila (Villarreal-Quintanilla y Encina-Domínguez, 2005). La topografía accidentada del área ha evitado el impacto antrópico en el bosque, sin embargo, actualmente se ha incrementado el peligro de que el fuego destruya esta comunidad por acumulación de material combustible (A. Nájera Díaz, com. pers.), por lo que es importante realizar investigación que permita implementar técnicas de manejo de combustibles, enfocadas a la conservación a largo plazo de este bosque, así como de las especies que ahí se presentan.

\section{Agradecimientos}

Agradecemos al herbario ANSM de la Universidad Autónoma Agraria Antonio Narro las facilidades brindadas para la realización del presente trabajo; a José A. Villarreal por su ayuda en la determinación de las herbáceas y a Socorro González E., Victoria Sosa y a un revisor anónimo, por sus valiosas sugerencias para mejorar la presentación; a Erika Reyna O., por la elaboración de la figura 1. 


\section{Literatura citada}

Aguirre-Calderón O., Jiménez-Pérez J., Kramer H. y Akça A. 2003. Análisis estructural de ecosistemas forestales en el Cerro del Potosí, Nuevo León, México. Ciencia-UANL 6: 219-225.

Alvarado D., de Bauer L. y Galindo J.A. 1991. Declinación y muerte de bosque de oyamel (Abies religiosa) en el sur del Valle de México. Agrociencia, serie Recursos Naturales Renovables 3: $123-143$.

Anónimo. 1983. Síntesis geográfica de Coahuila. México. Instituto Nacional de Estadística Geografía e Informática. Secretaría de Programación y Presupuesto. 163 pp.

Anónimo. 1996. Decreto del Área Natural Protegida, con Carácter de Zona Sujeta a Conservación Ecológica, un Área de la Serranía conocida como Zapalinamé. Periódico Oficial del Estado, Tomo CIII, No. 83, Saltillo, Coahuila.

Anónimo. 1998. Programa de Manejo de la Zona Sujeta a Conservación Ecológica "sierra de Zapalinamé". Universidad Autónoma Agraria Antonio Narro, Buenavista, Saltillo. Coahuila. $179 \mathrm{pp}$.

Arce G.L. y Marroquín J.S. 1985. Las unidades fisonómico-florísticas del cañón de San Lorenzo, Saltillo, Coahuila, México. Biótica 10: 369-393.

Ávila-Bello C.H., 2000. Ecología poblacional de Abies hickelii en el Pico de Orizaba, Veracruz, Colegio de Postgraduados. Informe Final SNIB-CONABIO proyecto No. L172. México D.F.

Ávila-Bello C. y López-Mata L. 2001. Distribución y análisis estructural de Abies hickelii (Flous et Gaussen) en México. Interciencia 26: 244-251.

Briones V.O. 1991. Sobre la flora, vegetación y fitogeografía de la sierra de San Carlos, Tamaulipas. Acta Botánica Mexicana 16: $15-44$

Bauer I. y Hernández T.T. 1986. Contaminación una amenaza para la vegetación en México. Colegio de Postgraduados. Chapingo, Estado de México. 84 pp.

Challenger A. 1998. Utilización y conservación de ecosistemas terrestres de México. Pasado, presente y futuro, Comisión Nacional Para la Conservación y uso de la Biodiversidad, México, D.F. 847 pp.

Cisneros-Lepe A. 2005. Estructura, composición florística y diversidad de los bosques de Abies en el estado de Jalisco, México. Tesis de Licenciatura. Universidad de Guadalajara. Autlán de Navarro Jalisco. $101 \mathrm{pp}$.

Cornejo-Oviedo E. 1987. Aspectos ecológicos y dasonómicos del bosque de Pseudotsuga-Pinus-Abies, en la sierra la Marta, Arteaga, Coahuila. Tesis de Licenciatura, Universidad Autónoma Agraria Antonio Narro. Buenavista, Saltillo, Coahuila. 196 pp.

Daniel T., Helmes J. y Baker F. 1982. Principios de silvicultura. Mc Graw Hill, México, D.F. 496 pp.

Farjon A. 1990. Pinaceae: drawings and descriptions of the genera Abies, Cedrus, Pseudolarix, Keteleeria, Nothotsuga, Tsuga, Cathaya, Pseudotsuga, Larix and Picea. Koeltz Scientific Books. Königstein

Figueroa-Rangel B. y M. Olvera-Vargas M. 2000. Dinámica de la composición de especies en bosques de Quercus crassipes Humb. et Bonpl. en cerro Grande, Sierra de Manantlán, México. Agrociencia, serie Recursos Naturales Renovables 1: 91-98.

Figueroa-Rangel B. y Moreno G.S. 1993. Estructura y sucesión en poblaciones de Abies religiosa (Kunt.) Schlecht. et Cham. y Abies religiosa var. emarginata Loock. et Martínez ex Martínez, de la sierra de Manantlán. Jalisco. Agrociencia, serie Re cursos Naturales Renovables 3: 49-63.

Franco-Pizaña J.G. 1990. Dinámica de la regeneración de Pseudotsuga flahaulti Flous, en el bosque de Pseudotsuga - Pinus - Abies. Tesis de Licenciatura, Universidad Autónoma Agraria Antonio Narro. Buenavista, Saltillo, Coahuila. 158 pp.

Galindo-Jaimes L., González-Espinosa M., Quintana-Asencio P. y García L. 2002. Tree composition and structure in disturbed stands with varying dominance by Pinus spp. in the highlands of Chiapas, México. Plant Ecology 162: 259-272.

Hernández-Martínez A. 1984. Estructura y regeneración del bosque natural de oyamel (Abies religiosa (HBK.) Schl. et Cham.) en el Cofre de Perote, Veracruz. Tesis de Licenciatura, Universidad Autónoma Agraria Antonio Narro. Buenavista, Saltillo, Coahuila.

Hocker H.M. 1984. Introducción a la biología forestal. FAO. Roma. 135 pp.

Jardel-Peláez E., Santiago-Pérez A.L. Cortés-Montaño C. y Castillo-Navarro F. 2004. Sucesión y dinámica de rodales. En: Cuevas R. y Fardel E.P. Eds. Flora y vegetación de la estación Científica Las Joyas. Petra ediciones. Autlán de Navarro, Jalisco, México.

Kormondy E.J. 1985. Conceptos de ecología. Alianza Universitaria. Madrid, España. 278 pp.

López-López M.A., Velásquez-Mendoza J., Velásquez-Mendoza A., González-Romero V. y Cetina-Alcalá V.M. 1998. Estado nutrimental de Abies religiosa en una área con problemas de contaminación ambiental. Agrociencia Serie Recursos Naturales Renovables. 1: 53-59.

Madrigal X. 1967. Contribución al conocimiento ecológico de los bosques de oyamel (Abies religiosa (Kunth.) Schl. et Cham.) en el Valle de México. Boletín Técnico 18. INIF. México, D.F.

Marroquín de la Fuente J.S. 1976. Vegetación y florística del noreste de México. I. Aspectos sinecológicos en Coahuila. Revista de la Sociedad Méxicana de Historia Natural 36: 69-101.

Medina B. R. 1983. Delimitación de sitios circulares de muestreo en investigación forestal. Ciencia Forestal 8: 3-25.

Meganck R.A. Carrera J.L., Rodríguez F.C. y Serrato V.C. 1981 Plan de manejo para el uso múltiple del cañón de San Lorenzo. Universidad Autónoma Agraria Antonio Narro. Organización de los Estados Americanos (OEA). Saltillo, Coahuila, México. 129 pp.

Mueller-Dombois D. y Ellenberg H. 1974. Aims and methods of vegetation ecology. John Wiley \& Sons. Inc., New York. 547 pp.

Nájera-Castro A. 2000. Efecto de tres tratamientos silvícolas sobre el microclima y la regeneración en un bosque de Abies-Pseudotsuga-Pinus, en Arteaga, Coahuila. Tesis de maestría en ciencias forestales. Universidad Autónoma Agraria Antonio Narro. Buenavista, Saltillo, Coahuila, México. 173 pp.

Narave-Flores H. 1985. Vegetación del Cofre de Perote, Veracruz, México. Biótica 10: 35-64.

Niembro-Rocas, A. 1986. Mecanismos de reproducción sexual en pinos. Limusa. México, D.F. 130 pp.

Olvera V.M. Moreno G.S. y Figueroa R.B. 1996. Sitios permanentes para la investigación silvícola. Manual para su establecimiento. Libros del Instituto Manantlán. Universidad de Guadalajara, Guadalajara, Jalisco, México. 55 pp.

Reyna-Olvera E. 1998. Evaluación de la regeneración natural de 


\section{ENCINA-DOMÍNGUEZ, ET AL.}

coníferas en el área incendiada en 1975, en la Sierra La Marta, Arteaga, Coahuila. Tesis de licenciatura. Universidad Autónoma Agraria Antonio Narro. Buenavista, Saltillo, Coahuila, México. $101 \mathrm{pp}$.

Rzedowski J. 1978. Vegetación de México. Ed. Limusa. México, D.F. 432 pp.

Rzedowski J. 1991. Diversidad y orígenes de la flora fanerogámica de México. Acta Botánica Mexicana 14: 3-21.

Rzedowski J. 1998. Diversidad y origen de la flora fanerogámica de México. En: Ramamoorthy, T.P., Bye R., Lot A. y Fa J. Eds. Diversidad biológica de México: Origen y distribución, pp. 129-145. Instituto de Biología, UNAM. México.

Rzedowski G.C de Rzedowski y colaboradores. 2001. Flora fanerogámica del Valle de México. 2a. ed., Instituto de Ecología, A.C. y Comisión Nacional para el Conocimiento y Uso de la Biodiversidad, Pátzcuaro, Michoacán. 1406 pp.

Saavedra-Romero L., Alvarado-Rosales D., Vargas-Hernández J. y Hernández-Tejeda T. 2003. Análisis de la precipitación pluvial en bosques de Abies religiosa (HBK.) Schlecht. et Cham., en el sur de la ciudad de México. Agrociencia serie Recursos Naturales Renovables 1: 57-64.

Recibido: 13 de agosto de 2008

Aceptado: 17 de octubre de 2008
Sánchez-González A., López-Mata L. y Granados Sánchez D. 2005. Semejanza florística entre los bosques de Abies religiosa (Kunth.) Cham. \& Schltdl. de la Faja Volcánica Transmexicana. Boletín del Instituto de Geografía UNAM 56: 62-76.

SEMARNAT [Secretaría del Medio Ambiente y Recursos Naturales]. 2002. Norma Oficial Mexicana NOM-059-SEMARNAT2001 que determina las especies nativas de México de flora y fauna silvestres - categorías de riesgo y especificaciones para su inclusión, exclusión o cambio - lista de especies en riesgo. Diario Oficial de la Federación, 2a sección, 6 de Marzo de 2002. México D. F. 40 pp.

Spurr S. y Barnes B. 1982. Ecología Forestal. AGT. Editor, S.A. México. $321 \mathrm{pp}$.

Verduzco-Gutiérrez J. 1976. Protección forestal. PATENA, A.C. Chapingo, México. 369 pp.

Villarreal-Quintanilla J.A. y Valdés-Reyna J. 1992-93. Vegetación de Coahuila, México. Revista de Manejo de Pastizales 6: 9-18.

Villarreal-Quintanilla J.A. y Encina-Domínguez J. 2005. Plantas vasculares endémicas de Coahuila y algunas áreas adyacentes, México. Acta Botánica Mexicana 70: 1-46. 
ESTRUCTURA Y FLORÍSTICA DEL BOSQUE DE OYAMEL, ZAPALINAMÉ

Anexo 1. Listado florístico de los bosques de oyamel de la sierra de Zapalinamé.

\begin{tabular}{|c|c|c|c|c|}
\hline DIVISIÓN - CLASE & FAMILIA & & & \\
\hline & ASPLENIACEAE & Asplenium & resiliens & Kunze \\
\hline \multirow[t]{2}{*}{ PTERIDOPHYTA } & POLYPODIACEAE & Pleopeltis & guttata & E.B. Andrews et Windham \\
\hline & PTERIDACEAE & $\begin{array}{l}\text { Cheilanthes } \\
\text { Pellaea }\end{array}$ & $\begin{array}{l}\text { tomentosa } \\
\text { ternifolia }\end{array}$ & $\begin{array}{l}\text { Link } \\
\text { (Cav.) Link }\end{array}$ \\
\hline \multirow[t]{2}{*}{ PINOPHYTA } & PINACEAE & $\begin{array}{l}\text { Abies } \\
\text { Pseudotsuga } \\
\text { Pinus } \\
\text { Pinus }\end{array}$ & $\begin{array}{l}\text { vejarii } \\
\text { menziesii } \\
\text { greggii } \\
\text { hartwegii }\end{array}$ & $\begin{array}{l}\text { Martínez } \\
\text { (Mirb.) Franco } \\
\text { Engelm. } \\
\text { Lundl. }\end{array}$ \\
\hline & CUPRESSACEAE & Cupressus & arizonica & Greene \\
\hline \multirow[t]{17}{*}{ MAGNOLIOPSIDA } & APIACEAE & $\begin{array}{l}\text { Donnellsmithia } \\
\text { Eryngium }\end{array}$ & $\begin{array}{l}\text { ternata } \\
\text { hemsleyanum }\end{array}$ & $\begin{array}{l}\text { (S. Watson) Mathias et Constance } \\
\text { H. Wolfin Engler }\end{array}$ \\
\hline & ASTERACEAE & $\begin{array}{l}\text { Achillea } \\
\text { Ageratina } \\
\text { Ageratina } \\
\text { Ageratina } \\
\text { Ageratina } \\
\text { Cirsium } \\
\text { Dahlia } \\
\text { Erigeron } \\
\text { Phanerostylis } \\
\text { Senecio } \\
\text { Senecio } \\
\text { Senecio } \\
\text { Senecio } \\
\text { Solidago } \\
\text { Tagetes }\end{array}$ & $\begin{array}{l}\text { millefolium } \\
\text { calophylla } \\
\text { nesomii } \\
\text { saltillensis } \\
\text { zapalinama } \\
\text { pringlei } \\
\text { coccinea } \\
\text { basilobatus } \\
\text { nesomii } \\
\text { coahuilensis } \\
\text { madrensis } \\
\text { richardsonii } \\
\text { zimapanicus } \\
\text { muelleri } \\
\text { lucida }\end{array}$ & $\begin{array}{l}\text { L. } \\
\text { (Blake) R.M. King et H. Rob. } \\
\text { B. L. Turner } \\
\text { (B.L. Rob.) R.M. King et H. Rob. } \\
\text { B. L. Turner } \\
\text { (S. Watson) Petrark. } \\
\text { Cav. } \\
\text { S.F. Blake } \\
\text { (B. L. Turner) R.M. King et H. Rob. } \\
\text { Greenm. } \\
\text { A. Gray } \\
\text { B.L. Turner } \\
\text { Hemsl. } \\
\text { Standl. } \\
\text { Cav. }\end{array}$ \\
\hline & BORAGINACEAE & Onosmodium & dondrantale & I. M. Johnston \\
\hline & BRASSICACEAE & Erysimum & capitatum & (Douglas) Greene \\
\hline & CAMPANULACEAE & Campanula & rotundifolia & L. \\
\hline & CAPRIFOLIACEAE & $\begin{array}{l}\text { Lonicera } \\
\text { Symphoricarpos }\end{array}$ & $\begin{array}{l}\text { pilosa } \\
\text { microphyllus }\end{array}$ & $\begin{array}{l}\text { (Kunth) Willd. } \\
\text { Kunth }\end{array}$ \\
\hline & CARYOPHYLLACEAE & $\begin{array}{l}\text { Arenaria } \\
\text { Arenaria }\end{array}$ & $\begin{array}{l}\text { lanuginosa } \\
\text { lycopodioides }\end{array}$ & $\begin{array}{l}\text { (Michx.) Rohrb. } \\
\text { Willd. }\end{array}$ \\
\hline & CELASTRACEAE & Paxistima & myrsinites & (Pursh.) Raf. \\
\hline & CORNACEAE & Cornus & stolonifera & Michx. \\
\hline & ERICACEAE & Arbutus & xalapensis & Kunth \\
\hline & EUPHORBIACEA & $\begin{array}{l}\text { Euphorbia } \\
\text { Euphorbia }\end{array}$ & $\begin{array}{l}\text { furcillata } \\
\text { brachycera }\end{array}$ & $\begin{array}{l}\text { Kunth } \\
\text { Engelm. }\end{array}$ \\
\hline & FABACEAE & $\begin{array}{l}\text { Lathyrus } \\
\text { Vicia }\end{array}$ & $\begin{array}{l}\text { parvifolius } \\
\text { ludoviciana }\end{array}$ & $\begin{array}{l}\text { S. Watson } \\
\text { Nutt. }\end{array}$ \\
\hline & FAGACEAE & $\begin{array}{l}\text { Quercus } \\
\text { Quercus } \\
\text { Quercus }\end{array}$ & $\begin{array}{l}\text { greggii } \\
\text { hintoniorum } \\
\text { saltillensis }\end{array}$ & $\begin{array}{l}\text { (A. DC.) Trel. } \\
\text { Nixon et C.H. Muller } \\
\text { Trel. }\end{array}$ \\
\hline & GARRYACEAE & Garrya & ovata & Benth. \\
\hline & GENTIANACEAE & Swertia & radiata & (Kell.) Kuntze \\
\hline & GERANIACEAE & Geranium & crenatifolium & H.E. Moore \\
\hline & HYDRANGEACEAE & Philadelphus & madrensis & Hemsl. \\
\hline
\end{tabular}


ENCINA-DomínGUEZ, ET AL.

\begin{tabular}{|c|c|c|c|c|}
\hline DIVISIÓN - CLASE & FAMILIA & & & \\
\hline & LAMIACEAE & $\begin{array}{l}\text { Salvia } \\
\text { Salvia } \\
\text { Salvia } \\
\text { Stachys }\end{array}$ & $\begin{array}{l}\text { regla } \\
\text { glechomifolia } \\
\text { grahamii } \\
\text { crenata }\end{array}$ & $\begin{array}{l}\text { Cav. } \\
\text { Kunth } \\
\text { Benth. } \\
\text { Raf. }\end{array}$ \\
\hline & OROBANCHACEAE & Conopholis & alpina & Liebm. \\
\hline & POLEMONIACEAE & Ipomopsis & aggregata & (Pursh.) V. Grant \\
\hline & POLYGONACEAE & Eriogonum & atrorubens & Engelm. \\
\hline & $\begin{array}{l}\text { PYROLACEAE } \\
\text { RANUNCULACEAE }\end{array}$ & $\begin{array}{l}\text { Chimaphila } \\
\text { Thalictrum }\end{array}$ & $\begin{array}{l}\text { umbellata } \\
\text { grandidentatum }\end{array}$ & $\begin{array}{l}\text { (L.) Nutt. } \\
\text { S. Watson }\end{array}$ \\
\hline & RHAMNACEAE & Rhamnus & betulifolia & Greene \\
\hline & ROSACEAE & $\begin{array}{l}\text { Holodiscus } \\
\text { Prunus }\end{array}$ & $\begin{array}{l}\text { discolor } \\
\text { serotina }\end{array}$ & $\begin{array}{l}\text { (Pursh) Maxim. } \\
\text { Ehrh. }\end{array}$ \\
\hline & SALICACEAE & $\begin{array}{l}\text { Populus } \\
\text { Salix }\end{array}$ & $\begin{array}{l}\text { tremuloides } \\
\text { paradoxa }\end{array}$ & $\begin{array}{l}\text { Michx. } \\
\text { Kunth }\end{array}$ \\
\hline & SCROPHULARIACEAE & Penstemon & campanulatus & (Cav.) Willd. \\
\hline & SOLANACEAE & $\begin{array}{l}\text { Nectouxia } \\
\text { Solanum }\end{array}$ & $\begin{array}{l}\text { formosa } \\
\text { verrucosum }\end{array}$ & $\begin{array}{l}\text { Kunth } \\
\text { Schltdl. }\end{array}$ \\
\hline \multirow[t]{4}{*}{ LILIOPSIDA } & IRIDACEAE & Sisyrinchium & scabrum & Cham. et Schltdl. \\
\hline & LILIACEAE & $\begin{array}{l}\text { Allium } \\
\text { Echeandia }\end{array}$ & $\begin{array}{l}\text { kunthii } \\
\text { flavescens }\end{array}$ & $\begin{array}{l}\text { G. Don } \\
\text { (Schult. et Schult) Cruden }\end{array}$ \\
\hline & ORCHIDACEAE & Malaxis & brachystachya & (Rchb.) Kuntze \\
\hline & POACEAE & $\begin{array}{l}\text { Bromus } \\
\text { Brachypodium } \\
\text { Festuca } \\
\text { Koeleria } \\
\text { Trisetum }\end{array}$ & $\begin{array}{l}\text { carinatus } \\
\text { mexicanum } \\
\text { valdesii } \\
\text { macrantha } \\
\text { deyeuxioides }\end{array}$ & $\begin{array}{l}\text { Hook. et Arn. } \\
\text { (Roem. et Schult.) Link } \\
\text { González - Ledesma } \\
\text { (Ledeb.) Schult. } \\
\text { (Kunth) Kunth }\end{array}$ \\
\hline
\end{tabular}

Anexo 2. Ubicación geográfica y datos ecológicos de los sitios de muestreo en el bosque de oyamel.

\begin{tabular}{ccccccc}
\hline Sitio & Latitud & Longitud & Altitud & $\begin{array}{c}\text { Pendiente }(\%) \\
\text { Temperatura }\left({ }^{\circ} \mathbf{C}\right)\end{array} \begin{array}{c}\text { Precipitación } \\
\text { media }(\mathbf{m m})\end{array}$ \\
\hline 1 & 25.3495 & 100.9059 & 2901 & 43 & 12 & 580 \\
2 & 25.3495 & 100.9079 & 2840 & 46 & 13 & 580 \\
3 & 25.3478 & 100.9047 & 3025 & 34 & 12 & 590 \\
4 & 25.3484 & 100.9028 & 2990 & 37 & 12 & 590 \\
5 & 25.3485 & 100.9099 & 2834 & 59 & 13 & 590 \\
6 & 25.3489 & 100.9118 & 2791 & 46 & 13 & 580 \\
7 & 25.3497 & 100.9136 & 2736 & 64 & 13 & 580 \\
8 & 25.3499 & 100.9159 & 2668 & 46 & 13 & 560 \\
9 & 25.3481 & 100.9074 & 2922 & 70 & 12 & 600 \\
10 & 25.3469 & 100.9075 & 2998 & 60 & 12 & 580 \\
\hline
\end{tabular}

